JOURNAL OF RESEARCH of the National Bureau of Standards-B. Mathematical Sciences
Vol. 80 B, No. 3, July-September 1976

\title{
A More Tractable Solution to a Singular Integral Equation Obtained by Solving a Related Hilbert Problem for Two Unknowns*
}

\author{
James A. Pennline**
}

(June 14, 1976)

The singular integral equation

$$
\int_{0}^{1}\left(\frac{1}{x-\sigma}+\frac{\frac{1}{2}}{x+\sigma}\right) \psi(x) d x=h, 0<\sigma<1,
$$

is converted into another singular integral equation

where

$$
\frac{1}{2} \frac{\int}{1}_{1}^{\infty} \frac{\phi(t)}{t-x} d t+\frac{3}{2} \sqrt{x}{\frac{\Gamma}{J_{1}}}^{\infty} \frac{\phi(t)}{\sqrt{t}(t-x)} d t=\frac{-2 h}{\sqrt{x}}, 1<x<\infty,
$$

$$
\phi(x)=\frac{\psi\left(\frac{1}{\sqrt{x}}\right)}{\sqrt{x}} .
$$

The former was derived from a boundary value problem in wave guide theory, and the solution is known. By solving a related Hilbert problem for two unknown functions, the solution of the latter integral equation is obtained. Then, the expression

$$
\psi(x)=\frac{1}{x} \phi\left(\frac{1}{x^{2}}\right)
$$

is compared to the former known expression obtained for $\psi(x)$, and the new expression is found to be more tractable.

Key words: Analytic function; Cauchy principle value; Hilbert problem for two unknowns; singular integral equation; wave-guide boundary problem.

\section{Introduction}

This paper illustrates an alternate procedure for solving in closed form an integral equation derived from a problem concerning a 3 -dimensional wave-guide structure and discussed by G. T. Bierman $[1]^{1}$ namely

$$
\int_{0}^{1}\left(\frac{1}{x-\sigma}+\frac{\frac{1}{2}}{x+\sigma}\right) \psi(x) d x=h, 0<\sigma<1
$$

The more general class of equations, of which (1) is a special case, in also treated by H. F. Bueckner [2] using numerical approximation methods.

One purpose for the alternate procedure is to arrive at the solution is a more simplified expression than previously obtained. Another purpose is also intended. The method we employ

AMS Subject Classification: Primary 45E05, 30A86; Secondary 78A50.

*An invited paper.

***Present address: Department of Mathematical Sciences, Virginia Commonwealth University, Richmond, VA 23284.

1 Figures in brackets indicate the literature references at the end of this paper. 
involves solving a Hilbert problem for two unknowns. Although the method for solving the general Hilbert problem for one unknown is known, [3], [5], [7], Vekua [10] states that the solution to the general problem for several unknowns cannot be expressed in closed form. The other purpose is to illustrate a special technique which can be used to solve certain nonhomogeneous Hilbert problems for several unknowns.

\section{Physical Origin of (1)}

Equation (1) has been shown by Bierman [1] to arise from a problem involving a waveguide structure in which a perfectly conducting surface extends from $-\infty$ to $\infty$ in the direction of the $z$ axis, making the boundary problem 2-dimensional in nature. Although some numerical results for the physical problem are reported in [6] and [9], the objective in [1] is to obtain a closed form solution. Here, we summarize the results beginning with the following diagram taken from [1] of the boundary and boundary conditions in the $x-y$ plane.

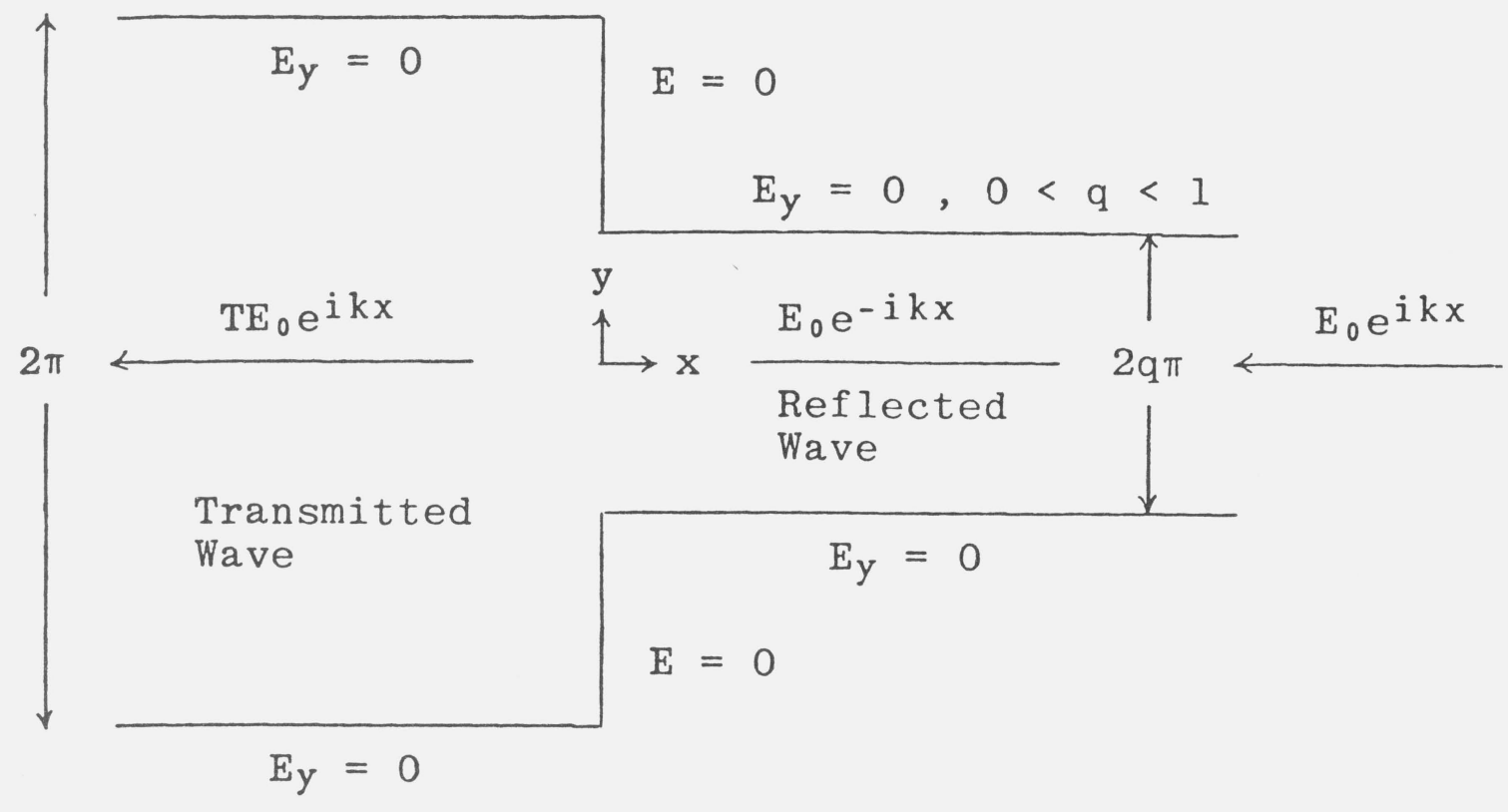

Figure 1. Widening, straight, 2-dimensional wave guide

By Fourier analysis, the following equation for $0 \leq y \leq q \pi$ is obtained.

$$
i k(1-\rho) E_{0}\left(q-\frac{1+\rho}{1-\rho}\right)=\frac{1}{\pi q} \int_{0}^{\mathrm{q} \pi} E^{\prime}(\xi)\left[\frac{\sin \left(\frac{\xi}{q}\right)}{\cos \left(\frac{\xi}{q}\right)-\cos \left(\frac{y}{q}\right)}+\frac{q \sin (\xi)}{\cos (\xi)-\cos (y)}\right] d \xi,
$$

where $E(x, y)$ is the $y$-component of the electric field of the wave guide, and $E(y)=E(0, y)$ represents the field in the aperture. Solving for $E^{\prime}(y)$ and hence $E(y)$ then determines the reflection coefficient $\rho$ by

$$
(1-\rho) E_{0}=\frac{1}{2 \pi q} \int_{-q \pi}^{q \pi} E(\xi) d \xi
$$

Once $\rho$ is computed, the transmission coefficient is given by

$$
T=q(1-\rho) .
$$

Although the general solution to (2) for arbitrary $q, 0<q<1$, is unknown, Bierman arrives at a solution for the specific case

$$
q=\frac{1}{2} .
$$


This is done by letting

$$
x=\cos (y), \sigma=\cos (\xi), \psi(x)=E^{\prime}\left(\cos ^{-1}(x)\right), h=\frac{\pi}{2} i k(1-\rho) E_{0}\left(\frac{1}{2}-\frac{1+\rho}{1-\rho}\right),
$$

in which case (2) for

$$
q=\frac{1}{2}
$$

becomes

$$
\int_{0}^{1}\left(\frac{1}{x-\sigma}+\frac{\frac{1}{2}}{x+\sigma}\right) \psi(x) d x=h, 0<\sigma<1
$$

Then (1) is solved for $\psi(x)$ and $E^{\prime}(y)$ obtained from (2c).

Actually, a general class of equations of which (1) is a specific case is treated in [1]. In the treatment, the general equation is converted into an equivalent integral equation to which the Wiener-Hopf technique is applied, i.e., the technique of Fourier transforms, functional equations, and analytic continuation procedures. The general solution obtained for (1) is

$$
\psi(x)=c_{0} k_{0}(x)+\frac{x h}{\pi i} \frac{d}{d x} \int_{0}^{1} \int_{x}^{1} k_{0}\left(\frac{x}{\xi}\right) k_{1}\left(\frac{\xi}{t}\right) \frac{1}{t} d \xi d t
$$

where

$$
k_{0}(x)=\frac{1}{\sqrt{\pi}} \frac{x^{-2 / 3}}{\sqrt{1-x^{2}}}\left[\left(1-{\sqrt{1-x^{2}}}^{2 / 3}+\left(1+{\sqrt{1-x^{2}}}^{2 / 3}\right], 0<x<1,\right.\right.
$$

and

$$
i \sqrt{\pi} k_{1}(x)=\left\{\begin{array}{l}
\frac{2 \pi}{3 \sqrt{3}}, 0<x<1 \\
-4 \int_{0}^{w}\left[\cosh \left(\frac{2}{3} \xi\right)+\frac{2}{3}\left(\frac{1-\xi^{2}}{1+\xi^{2}}\right) \sinh \left(\frac{2}{3} \xi\right)\right] \frac{\xi}{\left(\xi^{2}-1\right)^{2}} d \xi, w=x-\sqrt{x^{2}-1}, x>1 .
\end{array}\right.
$$

Expression $k_{0}(x)$ of $(3 a)$ is a nontrivial eigenfunction of $(2)$, and $c_{0}$ is a constant.

A disadvantage of the above result is the complicated expression for the particular part of the solution to (1). Limitation due to the difficulty in evaluating $k_{1}(x)$ and the particular part of the solution (3) is admitted in [1] by stating that it does not seem likely they can be expressed in terms of elementary functions. However, we shall show that the solution can be expressed in terms of elementary functions.

\section{An Alternate Approach to Solving (1)}

In this section, we shall convert (1) into a singular integral equation in which only the Cauchy kernel term $\frac{1}{t-x}$ appears under the integral sign. This new integral equation will subsequently be converted into an equivalent Hilbert problem for two unknown functions [10].

By the transformations,

$$
x=\frac{1}{r}, \sigma=\frac{1}{s},
$$

eq (1) becomes

$$
-s{\frac{\int}{\mathrm{J}_{1}}}^{\infty} \frac{\psi\left(\frac{1}{r}\right)}{r} \frac{1}{r-s} d r+\frac{s}{2} \int_{1}^{\infty} \frac{\psi\left(\frac{1}{r}\right)}{r} \frac{1}{r+s} d r=h, 1<s<\infty,
$$


where we have used a bar through the first integral sign to denote the Cauchy-principle value of the integral. Then the substitutions $s=\sqrt{x}, r=\sqrt{t}$, transform (4) into

$$
-\sqrt{x}{\frac{\int}{J_{1}}}_{1}^{\infty} \frac{\phi(t)}{(\sqrt{t}-\sqrt{x})} \frac{d t}{2 \sqrt{t}}+\frac{\sqrt{x}}{2} \int_{1}^{\infty} \frac{\phi(t)}{(\sqrt{t}+\sqrt{x})} \frac{d t}{2 \sqrt{t}}=h,
$$

where

$$
\phi(t)=\frac{\psi\left(\frac{1}{\sqrt{t}}\right)}{\sqrt{t}}
$$

Now if the term

$$
\frac{\sqrt{x}}{2} \int_{1}^{\infty} \frac{\phi(t)}{(\sqrt{t}-\sqrt{x})} \frac{d t}{2 \sqrt{t}}
$$

is added and subtracted on the left-hand side of $(5 \mathrm{a})$, then

$$
-\left(1+\frac{1}{2}\right) \sqrt{x}{\frac{\int}{J_{1}}}_{1}^{\infty} \frac{\phi(t)}{(\sqrt{t}-\sqrt{x})} \frac{d t}{2 \sqrt{t}}+\frac{1}{2} \sqrt{x}{\frac{\int}{J_{1}}}^{\infty} \frac{\phi(t)}{t-x} d t=h .
$$

Also, if the term

$$
\sqrt{x} \int_{1}^{\infty} \frac{\phi(t)}{(\sqrt{t}+\sqrt{x})} \frac{d t}{2 \sqrt{t}}
$$

is added and subtracted on the left-hand side of (5a), then

$$
-\sqrt{x} \frac{\int}{J}_{1}^{\infty} \frac{\phi(t)}{t-x} d t+\left(1+\frac{1}{2}\right) \sqrt{x} \int_{1}^{\infty} \frac{\phi(t)}{(\sqrt{t}+\sqrt{x})} \frac{d t}{2 \sqrt{t}}=h .
$$

Finally, by adding eqs (6) and (7),

$$
\frac{1}{2} \frac{\Gamma}{J}_{1}^{\infty} \frac{\phi(t)}{t-x} d t+\frac{3}{2} \sqrt{x}{\frac{\Gamma}{J_{1}}}_{1}^{\infty} \frac{\phi(t)}{\sqrt{t}(t-x)} d t=\frac{-2 h}{\sqrt{x}}, 1<x<\infty
$$

where

$$
\phi(x)=\frac{\psi\left(\frac{1}{\sqrt{x}}\right)}{\sqrt{x}}
$$

Equation (8) is a special case of the singular integral equation

$$
a(x){\frac{\Gamma}{J_{1}}}^{\infty} \frac{\phi(t)}{t-x} d t+b(x) \frac{\Gamma}{J}_{1}^{\infty} \frac{\phi(t)}{\sqrt{t}(t-x)} d t=c(x), 1<x<\infty
$$

where the bar through each integral sign denotes the Cauchy principle value of the integral. This class of equations is considered in [8] where the approach is to consider a Hilbert problem derived from (10). Another case of (10) has been shown by Latta [4] to arise from a convolution integral equation with finite limits. In the next section we begin discussing the Hilbert problem related to $(8)$.

\section{Conversion of (8) to a Hilbert Problem for Two Unknowns}

To solve (8), one can try to proceed with a method similar to one of the standard approaches used to solve certain singular integral equations with the Cauchy kernel term $\frac{1}{t-x}$ [3], [5], [7]. The method is based on the theory of Cauchy integrals [5], [7]. 
If a solution is known to exist, the theory of Cauchy integrals suggests its derivation (for reasons which will become clear in the ensuing analysis) by considering the functions

$$
F(z)=\frac{1}{2 \pi i} \int_{1}^{\infty} \frac{\phi(t)}{t-z} d t, G(z)=\frac{1}{2 \pi i} \int_{1}^{\infty} \frac{\phi(t)}{\sqrt{t}(t-z)} d t, z \epsilon[1, \infty) .
$$

If $\phi(t)$ is of a suitable class of functions, ${ }^{2}$ then $F(z)$ and $G(z)$ have the following properties:

I. $F(z)$ and $G(z)$ are analytic in the finite complex z-plane excluding the real semiinfinite interval $[1, \infty)$.

II. $F(z)$ and $G(z)$ have at worst growth rate at $z=1$ less than that of a simple pole.

III. There exists a $\gamma>0$ such that

and

$$
F(z)=0\left(|z|^{-\gamma}\right)
$$

$$
G(z)=0\left(|z|^{-\left(\gamma+\frac{1}{2}\right)}\right) \text { as }|z| \rightarrow \infty, z \epsilon[1, \infty) .
$$

Other basic properties of $F(z)$ and $G(z)$ come from relations between their boundary values. Let $F_{+}(x), G_{+}(x)$ and $F_{-}(x), G_{-}(x)$ denote the limits of $F(z), G(z)$ as $z \rightarrow x, 1<x<\infty$, from above and below the real axis respectively. From the theory of Cauchy integrals [5], [7], these limits exist and are given by ${ }^{3}$

$$
F_{ \pm}(x)=\frac{1}{2 \pi i}{\frac{\Gamma}{J_{1}}}_{1}^{\infty} \frac{\phi(t)}{t-x} d t \pm \frac{1}{2} \phi(x), 1<x<\infty, G_{ \pm}(x)=\frac{1}{2 \pi i}{\frac{\Gamma}{J_{1}}}_{1}^{\infty} \frac{\phi(t)}{\sqrt{t}(t-x)} d t \pm \frac{1}{2} \frac{\phi(x)}{\sqrt{x}}, 1<x<\infty
$$

which leads to the Plemelj formulae

$$
\begin{gathered}
F_{+}(x)+F_{-}(x)=\frac{1}{\pi i}{\frac{\int}{J_{1}}}^{\infty} \frac{\phi(t)}{t-x} d t, \\
F_{+}(x)-F_{-}(x)=\phi(x), \\
G_{+}(x)+G_{-}(x)=\frac{1}{\pi i} \int_{1}^{\infty} \frac{\phi(t)}{\sqrt{t}(t-x)} d t, \\
G_{+}(x)-G_{-}(x)=\frac{\phi(x)}{\sqrt{x}},
\end{gathered}
$$

$1<x<\infty$.

Now, the integral equation (8) can be expressed as an equivalent problem in terms of $F(z)$ and $G(z)$ defined in (11). Note that (13a) and (13c) reveal that (8) is equivalent to

$$
\frac{1}{2} \pi i\left(F_{+}(x)+F_{-}(x)\right)+\frac{3}{2} \sqrt{x} \pi i\left(G_{+}(x)+G_{-}(x)\right)=\frac{-2 h}{\sqrt{x}}, 1<x<\infty,
$$

${ }^{2}$ It is sufficient that $-\sqrt{x} \psi(\sqrt{x})$ be of class $C(A)$ described by Levinson [5] where $C=(0,1)$. Then, by results in [5], the function

$$
\Psi(z)=\frac{1}{2 \pi i} \int_{0}^{1} \frac{-\sqrt{t} \psi(\sqrt{t})}{t-z} d t
$$

is analytic in the complex $z$-plane cut along [0,1], and has at worst growth rate at $z=0$ and $z=1$ less than that of a simple pole. Thus, by replacing

$$
z \text { by } \frac{1}{z} \text { and } t \text { by } \frac{1}{t} \text { in } \Psi(z),
$$

conditions I, II, and III follow for the function

$$
F(z)=\frac{\Psi\left(\frac{1}{z}\right)}{z}=\frac{1}{2 \pi i} \int_{1}^{\infty} \frac{\psi\left(\frac{1}{\sqrt{t}}\right)}{\sqrt{t}}\left(\frac{d t}{t-z}\right) d t .
$$

${ }^{3}$ Again, it is sufficient that $--\sqrt{x} \psi(\sqrt{x})$ be in class $C(\mathrm{~A})$ described by Levinson [5] where $C=(0,1)$. 
while $(13 \mathrm{~b})$ and $(13 \mathrm{~d})$ reveal that

$$
F_{+}(x)-F_{-}(x)=\sqrt{x}\left(G_{+}(x)-G_{-}(x)\right), 1<x<\infty .
$$

Equations (14a) and (14b) along with conditions I, II, and III constitute a Hilbert problem for two unknown functions $F(z)$ and $G(z)$ [10]. Solution of the problem determines $\phi(x)$ to $(8)$ by either (13b) or (13d). In matrix form, (14a) and (14b) can be written as

$$
\left[\begin{array}{l}
F_{+}(x) \\
G_{+}(x)
\end{array}\right]-\left[\begin{array}{cc}
\frac{1}{2} \frac{-3}{2} \sqrt{x} \\
\frac{-1}{2 \sqrt{x}} & \frac{-1}{2}
\end{array}\right]\left[\begin{array}{l}
F_{-}(x) \\
G_{-}(x)
\end{array}\right]=\frac{1}{\pi i}\left(\frac{-2 h}{\sqrt{x}}\right)\left[\begin{array}{c}
\frac{1}{2} \\
\frac{1}{2 \sqrt{x}}
\end{array}\right], 1<x<\infty,
$$

which is the matrix analogue of the Hilbert problem on an arc for one unknown function [5], [7].

As mentioned in the introduction, the method for solving the general Hilbert problem for one unknown is known. However, as Vekua [10] states, the solution to the general Hilbert problem for several unknowns cannot be expressed in closed form. One of two special methods developed in [8] will be used to solve the Hilbert problem (14).

\section{Solution of the Hilbert Problem (14)}

Relations (14a) and (14b) do not uncouple into equations involving just boundary values of $F$ or just boundary values of $G$. To solve this system, a special procedure which generates particular solutions to certain nonhomogeneous problems from known homogeneous solutions will be used.

We first observe that the eigenfunction (3a) to (1) admits an eigenfunction to the corresponding homogeneous form of (8). Specifically, by the relation (9) and the expression (3a), the function

$$
\phi_{0}(x)=\frac{\sqrt{\pi} k_{0}\left(\frac{1}{\sqrt{x}}\right)}{\sqrt{x}}=\frac{1}{\sqrt{x-1}}\left[(\sqrt{x}-\sqrt{x-1})^{2 / 3}+(\sqrt{x}+\sqrt{x-1})^{2 / 3}\right]
$$

must be an eigenfunction and the only nontrivial one satisfying

$$
\frac{1}{2}{\frac{\int_{1}}{J_{1}}}^{\infty} \frac{\phi_{0}(t)}{t-x} d t+\frac{3}{2} \sqrt{x}{\frac{\int^{\infty}}{J_{1}}}^{\infty} \frac{\phi_{0}(t)}{\sqrt{t}(t-x)} d t=0,1<x<\infty .
$$

Likewise, by the Plemelj formulae (13) and the integral definitions (11), the function

$$
\left[\begin{array}{l}
F_{0}(z) \\
G_{0}(z)
\end{array}\right]=\left[\begin{array}{c}
\frac{1}{2 \pi i} \int_{1}^{\infty} \frac{\phi_{0}(t)}{t-z} d t \\
\frac{1}{2 \pi i} \int_{1}^{\infty} \frac{\phi_{0}(t)}{\sqrt{t}(t-z)} d t
\end{array}\right], z \notin[1, \infty),
$$

must be an eigenfunction to

$$
\begin{gathered}
\frac{1}{2} \pi i\left(F_{0_{+}}(x)+F_{0_{-}}(x)\right)+\frac{3}{2} \sqrt{x} \pi i\left(G_{0_{+}}(x)+G_{0_{-}}(x)\right)=0, \\
\left(F_{0_{+}}(x)-F_{0_{-}}(x)\right)=\sqrt{x}\left(G_{0_{+}}(x)-G_{0_{-}}(x)\right), 1<x<\infty .
\end{gathered}
$$

To generate candidates for a particular solution to (14), we shall also need the eigenvector

$$
\left[\begin{array}{c}
\tilde{F}_{0}(z) \\
\tilde{G}_{0}(z)
\end{array}\right]
$$


satisfying the Hilbert problem "adjoint" to (18), i.e.,

$$
\begin{gathered}
\frac{3}{2} \pi i\left(\tilde{F}_{0_{+}}(x)+\tilde{F}_{0_{-}}(x)\right)+\frac{1}{2} \sqrt{x} \pi i\left(\tilde{G}_{0_{+}}(x)+\tilde{G}_{0_{-}}(x)\right)=0, \\
\tilde{F}_{0_{+}}(x)-\tilde{F}_{0_{-}}(x)=\sqrt{x}\left(\tilde{G}_{0_{+}}(x)-\tilde{G}_{0_{-}}(x)\right), 1<x<\infty .
\end{gathered}
$$

The Hilbert problem (19), is of course, derived from the singular integral equation adjoint to (16), i.e.,

$$
\frac{3}{2}{\frac{\int}{J_{1}}}^{\infty} \frac{\tilde{\phi}_{0}(t)}{t-x} d t+\frac{1}{2} \sqrt{x}{\frac{\Gamma}{J_{1}}}^{\infty} \frac{\tilde{\phi}_{0}(t)}{\sqrt{t}(t-x)} d t=0,1<x<\infty
$$

by applying the Plemelj formulae for Cauchy type integrals to

$$
\left[\begin{array}{l}
\tilde{F}_{0}(z) \\
\tilde{G}_{0}(z)
\end{array}\right]=\left[\begin{array}{l}
\frac{1}{2 \pi i} \int_{1}^{\infty} \frac{\tilde{\phi}_{0}(t)}{t-z} d t \\
\frac{1}{2 \pi i} \int_{1}^{\infty} \frac{\tilde{\phi}_{0}(t)}{\sqrt{t}(t-z)} d t
\end{array}\right]
$$

By the same procedure in which (8) was derived from (1), (20) can be derived from the integral equation

$$
\int_{0}^{1}\left(\frac{1}{x-\sigma}+\frac{-\frac{1}{2}}{x+\sigma}\right) \tilde{\psi}_{0}(x) d x=0,0<\sigma<1
$$

where

$$
\tilde{\phi}_{0}(x)=\frac{\tilde{\psi}_{0}\left(\frac{1}{\sqrt{x}}\right)}{\sqrt{x}}
$$

But (22) can be solved by the method in [1] which shows that there is one nontrivial eigenfunction given by

$$
\tilde{\psi}_{0}(x)=\frac{x^{-1 / 3}}{\sqrt{1-x^{2}}}\left[\left(1-\sqrt{1-x^{2}}\right)^{1 / 3}+\left(1+\sqrt{1-x^{2}}\right)^{1 / 3}\right]
$$

Hence,

$$
\tilde{\phi}_{0}(x)=\frac{\tilde{\psi}_{0}\left(\frac{1}{\sqrt{x}}\right)}{\sqrt{x}}=\frac{1}{\sqrt{x-1}}\left[(\sqrt{x}-\sqrt{x-1})^{1 / 3}+(\sqrt{x}+\sqrt{x-1})^{1 / 3}\right],
$$

and therefore, $\tilde{F}_{0}(z)$ and $\tilde{G}_{0}(z)$ in $(21)$ are known.

Now, in order to explain a method in [8] which will construct a candidate for a particular solution to (14), we are going to illustrate the idea it involves on a simple Hilbert problem for one unknown. This will require less mechanics than if it is explained directly on (14).

Consider the function

$$
h(z)=\frac{1}{\sqrt{z-1}}
$$

which is analytic in the $z$-plane cut from $z=1$ to $z=\infty$ along the real axis. With the choice of the branch

$$
\sqrt{z-1}=\sqrt{r} e^{i \theta / 2}, 0<\theta<2 \pi,
$$




$$
h_{ \pm}(x)=\frac{1}{ \pm \sqrt{x-1}}, 1<x<\infty
$$

where $h_{+}(x)$ and $h_{-}(x)$ are limits of $h(z)$ as $z \rightarrow x, 1<x<\infty$, from above and below the cut respectfully. Hence $h(z)$ is a solution to the homogeneous Hilbert problem

$$
f_{+}(x)+f_{-}(x)=0,1<x<\infty,
$$

where $f(z)$ is required to be analytic in the $z$-plane cut from $z=1$ to $z=\infty$ along the real axis, to tend to zero algebraically as $|z| \rightarrow \infty$, and to have at worst growth rate at $z=1$ less than that of a simple pole. Also, $b_{0} h(z)$ for any constant $b_{0}$ is a solution to $(* 3)$.

Whole families of particular solutions to certain nonhomogeneous Hilbert problems can be generated from $h(z)$ in the following way. Let $z_{0}$ be a point in the $z$-plane not on the real axis from $z=1$ to $z=\infty$. From

$$
\frac{h(z)}{\left(z-z_{0}\right)^{n}}
$$

subtract the principle part of the Laurent expansion of

$$
\frac{h(z)}{\left(z-z_{0}\right)^{n}},
$$

about $z=z_{0}$, and define $f_{n}(z)$ as

$$
f_{n}(z)=\frac{h(z)}{\left(z-z_{0}\right)^{n}}-\frac{h\left(z_{0}\right)}{\left(z-z_{0}\right)^{n}}-\sum_{k=1}^{n-1} \frac{h^{(k)}\left(z_{0}\right)}{k !} \frac{1}{\left(z-z_{0}\right)^{n-k}},
$$

where $h^{(k)}\left(z_{0}\right)$ is $k$ th derivative of $h$ evaluated at $z_{0}$. For $n=1,2, \ldots$, a countably infinite family of functions is constructed. Each member is analytic in the $z$-plane cut from $z=1$ to $z=\infty$ along the real axis. let

To see how the functions (*4) form solutions to certain nonhomogeneous Hilbert problems,

$$
\sum_{k=1}^{\infty} \lambda_{k} f_{k}(z)
$$

represent a constant coefficient linear combination of functions defined by $(* 4)$. If this linear combination converges uniformly to a function $g(z)$ at all finite $z$ in the cut plane and to $g_{+}(x)$, $g_{-}(x)$ on each respective side of the cut, then $g(z)$ will be a particular solution to the Hilbert problem

where

$$
f_{+}(x)+f_{-}(x)=\sum_{k=1}^{\infty} h_{k}(x), 1<x<\infty,
$$

$$
h_{k}(z)=f_{k}(z)-\frac{h(z)}{\left(z-z_{0}\right)^{k}}, k=1,2 \ldots
$$

Conversely, if given the Hilbert problem

$$
f_{+}(x)+f_{-}(x)=\sum_{k=1}^{m} \frac{b_{k}}{\left(x-z_{0}\right)^{k}}, 1<x<\infty,
$$

where $b_{k}, k=1,2, \ldots, \ldots, m$ are constants, there is a constant coefficient linear combination of functions from $(* 4)$, i.e.,

$$
\sum_{k=1}^{m} \lambda_{k} f_{k}(z)
$$

which satisfies $\left({ }^{*} 7\right)$. The constants $\lambda_{k}, k=1,2, \ldots, \ldots$, can be determined by substituting the boundary values of $(* 8)$ into $(* 7)$ and equating coefficients of like powers of

$$
\frac{1}{x-z_{0}} \text {. }
$$


The generating function scheme illustrated above can be applied to the Hilbert problem (14) to find a particular solution. We have as known homogeneous solutions the eigenvector (17) to the problem (18) and the eigenvector (21) to the problem (19). There are several ways to generate families of particular solutions to certain nonhomogeneous problems with left-hand side as in (14).

By noting the discussion of the scalar Hilbert problem $(* 7)$, it seems as though the following construction of functions will yield a particular solution to (14):

$$
\left[\begin{array}{c}
\frac{F_{0}(z)}{z} \\
\frac{G_{0}(z)}{z}
\end{array}\right]-\left[\begin{array}{c}
\frac{F_{0}(0)}{z} \\
\frac{G_{0}(0)}{z}
\end{array}\right],\left[\begin{array}{c}
\frac{F_{0}(z)}{z^{2}} \\
\frac{G_{0}(z)}{z^{2}}
\end{array}\right]-\left[\begin{array}{c}
\frac{F_{0}(0)}{z^{2}} \\
\frac{G_{0}(0)}{z^{2}}
\end{array}\right]-\left[\begin{array}{c}
\frac{F_{0}^{\prime}(0)}{z} \\
\frac{G_{0}^{\prime}(0)}{z}
\end{array}\right], \ldots,
$$

where $F_{0}(z)$ and $G_{0}(z)$ are defined in (17).

Another family of particular solutions can be formed as follows:

$$
\left[\begin{array}{l}
F_{1}(z) \\
G_{1}(z)
\end{array}\right]=\left[\begin{array}{l}
G_{0}(z) \\
\frac{F_{0}(z)}{z}-\frac{F_{0}(0)}{z}
\end{array}\right],\left[\begin{array}{l}
F_{n+1}(z) \\
G_{n+1}(z)
\end{array}\right]=\left[\begin{array}{l}
G_{n}(z) \\
\frac{F_{n}(z)}{z}-\frac{F_{n}(0)}{z}
\end{array}\right], n=1,2, \ldots
$$

However, direct substitution will verify that no constant multiple of the first vector in either of the above families will satisfy the boundary relation (14b).

An appropriate candidate for a particular solution to (14) actually comes from the following generation of functions:

$$
\left[\begin{array}{l}
F_{1}(z) \\
G_{1}(z)
\end{array}\right]=\left[\begin{array}{l}
\tilde{G}_{0}(z) \\
\frac{\tilde{F}_{0}(z)}{z}-\frac{\tilde{F}_{0}(0)}{z}
\end{array}\right],\left[\begin{array}{l}
F_{n+1}(z) \\
G_{n+1}(z)
\end{array}\right]=\left[\begin{array}{l}
G_{n}(z) \\
\frac{F_{n}(z)}{z}-\frac{F_{n}(0)}{z}
\end{array}\right], n=1,2, \ldots
$$

where $\tilde{F}_{0}(z)$ and $\tilde{G}_{0}(z)$ are defined in $(21)$. We will demonstrate that a particular solution to the Hilbert problem (14) is a specific constant multiple of

$$
\left[\begin{array}{l}
F_{1}(z) \\
G_{1}(z)
\end{array}\right]=\left[\begin{array}{l}
\tilde{G}_{0}(z) \\
\frac{\tilde{F}_{0}(z)}{z}-\frac{\tilde{F}_{0}(0)}{z}
\end{array}\right]
$$

The constant $\tilde{F}_{0}(0)$ is described by

$$
\tilde{F}_{0}(0)=\frac{1}{2 \pi i} \int_{1}^{\infty} \frac{\tilde{\phi}_{0}(t)}{t} d t
$$

where $\tilde{\phi}_{0}(t)$ is defined by $(24)$. This definite integral is elementary and not difficult to evaluate if one begins with the substitution $t=\cosh ^{2}(\theta)$. It turns out that

$$
\tilde{F}_{0}(0)=\frac{2}{\sqrt{3} i}
$$

We shall now show that some constant multiple of (25) is a particular solution of (14). Observe that by the way $F_{1}(z)$ and $G_{1}(z)$ were constructed, a constant multiple of $(25)$ satisfies conditions I, II, and III for a solution to (14). So, all that is needed is to make it fit the boundary relations (14a) and (14b). In terms of $\tilde{F}_{0}(z)$ and $\tilde{G}_{0}(z)$, the Plemelj formulae (13) for $F_{1}(z)$ and $G_{1}(z)$ in $(25)$ are

$$
\begin{gathered}
F_{1_{+}}(x)-F_{1_{-}}(x)=\frac{\tilde{\phi}_{0}(x)}{\sqrt{x}}, \\
F_{1_{+}}(x)+F_{1_{-}}(x)=\tilde{G}_{0_{+}}(x)+\tilde{G}_{0_{-}}(x),
\end{gathered}
$$




$$
\begin{gathered}
G_{1_{+}}(x)-G_{1_{-}}(x)=\frac{1}{x} \tilde{\phi}_{0}(x), \\
G_{1_{+}}(x)+G_{1_{-}}(x)=\frac{\tilde{F}_{0_{+}}(x)+\tilde{F}_{0_{-}}(x)}{x}-\frac{2 \tilde{F}_{0}(0)}{x}, 1<x<\infty .
\end{gathered}
$$

First, note that any constant multiple, $\lambda$, of (25) satisfies the second relation (14b), i.e.,

$$
\lambda\left(F_{1_{+}}(x)-F_{1_{-}}(x)\right)=\lambda \sqrt{x}\left(G_{1_{+}}(x)-G_{1_{-}}(x)\right), 1<x<\infty .
$$

In order for

$$
\lambda\left[\begin{array}{l}
F_{1}(z) \\
G_{1}(z)
\end{array}\right]
$$

to satisfy relation (14a), $\lambda$ must be chosen so that

$$
\frac{1}{2} \pi i \lambda\left(F_{1_{+}}(x)+F_{1_{-}}(x)\right)+\frac{3}{2} \sqrt{x} \pi i \lambda\left(G_{1_{+}}(x)+G_{1_{-}}(x)\right)=\frac{-2 h}{\sqrt{x}}, 1<x<\infty .
$$

By substituting the boundary relations for $F_{1}(z)$ and $G_{1}(z)$ in $(27),(28 \mathrm{~b})$ becomes

$$
\lambda\left[\frac{1}{2} \pi i\left(\tilde{G}_{0_{+}}(x)+\tilde{G}_{0_{-}}(x)\right)+\frac{3}{2} \frac{\pi i}{\sqrt{x}}\left(\tilde{F}_{0_{+}}(x)+\tilde{F}_{0-}(x)\right)\right]+\frac{3}{2} \sqrt{x} \pi i \lambda\left(\frac{-2 \tilde{F}_{0}(0)}{x}\right)=\frac{-2 h}{\sqrt{x}} .
$$

By the known relation (19), (29) reduces to

$$
\frac{3}{2} \sqrt{x} \pi i \lambda\left(\frac{-2 \tilde{F}_{0}(0)}{x}\right)=\frac{-2 h}{\sqrt{x}} .
$$

Finally, by substituting (26) into (30) and solving for $\lambda$,

$$
\lambda=\frac{h}{\pi \sqrt{3}} \cdot
$$

What has just been shown is that the vector

$$
\frac{h}{\pi \sqrt{3}}\left[\begin{array}{l}
F_{1}(z) \\
G_{1}(z)
\end{array}\right]
$$

is a particular solution to the Hilbert problem (14). The general solution to (14) is an arbitrary constant multiple of the homogeneous solution (17) plus a particular solution. Therefore, the general solution to (14) is given by

$$
\left[\begin{array}{l}
F(z) \\
G(z)
\end{array}\right]=a_{0}\left[\begin{array}{l}
F_{0}(z) \\
G_{0}(z)
\end{array}\right]+\frac{h}{\pi \sqrt{3}}\left[\begin{array}{l}
F_{1}(z) \\
G_{1}(z)
\end{array}\right]
$$

where $F_{0}(z)$ and $G_{0}(z)$ are given by (17).

\section{A More Tractable Solution to (1)}

The general solution to the singular integral equation (8) is now readily obtained from (31). By the Plemelj formulae (13b), the general solution to ( 8 ) is given by

$$
\begin{aligned}
\phi(x) & =F_{+}(x)-F_{-}(x) \\
& =a_{0}\left(F_{0_{+}}(x)-F_{0-}(x)\right)+\frac{h}{\pi \sqrt{3}}\left(F_{1_{+}}(x)-F_{1_{-}}(x)\right)
\end{aligned}
$$




$$
=a_{0} \phi_{0}(x)+\frac{h}{\pi \sqrt{3}}\left(\frac{\tilde{\phi}_{0}(x)}{\sqrt{x}}\right), 1<x<\infty
$$

where $\phi_{0}(x)$ is given by (15) and $\tilde{\phi}_{0}(x)$ is given by (24).

In turn, by the relations (9), (15), and (22a), the general solution $\psi(x)$ to (1) is given by

$$
\psi(x)=a_{0} \sqrt{\pi} k_{0}(x)+\frac{h x}{\pi \sqrt{3}} \tilde{\psi}_{0}(x), 0<x<1,
$$

where $k_{0}(x)$ is given by $(3 a)$ and $\tilde{\psi}_{0}(x)$ is given by $(23)$. This expression for $\psi(x)$ is certainly a more tractable one than the expression for $\psi(x)$ given by (3).

This result can now be used to finish the analysis of the wave-guide problem. An identification in $(2 \mathrm{c})$ says that

$$
E^{\prime}(y)=\psi(\cos (y))=a_{0} \sqrt{\pi} k_{0}(\cos (y))+\frac{h}{\pi \sqrt{3}} \cos (y) \tilde{\psi}_{0}(\cos (y)) .
$$

Let

$$
\sqrt{\pi} k_{0}(\cos (y))=\psi_{0}(\cos (y))
$$

so that

$$
E^{\prime}(y)=a_{0} \psi_{0}(\cos (y))+\frac{h}{\pi \sqrt{3}} \cos (y) \tilde{\psi}_{0}(\cos (y))
$$

Following the analysis of Bierman [1], $E^{\prime}(y)$ is odd and continuous at $y=0$. Therefore, $E^{\prime}(0)=0$, or equivalently

$$
\lim _{y \rightarrow 0}\left(a_{0} \psi_{0}(\cos (y))+\frac{h}{\pi \sqrt{3}} \cos (y) \tilde{\psi}_{0}(\cos (y))\right)=0 .
$$

Relation (35) thus determines $a_{0}$ uniquely,

$$
a_{0}=\frac{-h}{\pi \sqrt{3}}
$$

To obtain $E(y)$, eq (34) is integrated, and the constant of integration is chosen so that

$$
E\left(\frac{\pi}{2}\right)=0
$$

as required in [1].

$$
E(y)=\frac{h}{\pi \sqrt{3}} \int_{y}^{\pi / 2}\left(\cos (\sigma) \tilde{\psi}_{0}(\cos (\sigma))-\psi_{0}(\cos (\sigma))\right) d \sigma
$$

The reflection coefficient, $\rho$, is obtained from (2a).

$$
\begin{aligned}
(1-\rho) E_{0}=\frac{2}{\pi} \int_{0}^{\pi / 2} E(\xi) d \xi & =\frac{2}{\pi} \frac{h}{\pi \sqrt{3}} \int_{0}^{\pi / 2} \int_{\xi}^{\pi / 2}\left(\cos (\sigma) \tilde{\psi}_{0}(\cos (\sigma))-\psi_{0}(\cos (\sigma))\right) d \sigma d \xi \\
& =\frac{-1}{2 \pi \sqrt{3}} i k E_{0}(1+3 \rho) \int_{0}^{\pi / 2}\left(\cos (\sigma) \tilde{\psi}_{0}(\cos (\sigma))-\psi_{0}(\cos (\sigma))\right) \sigma d \sigma .
\end{aligned}
$$


Hence

$$
\rho=\frac{1+M}{1-3 M}
$$

where

$$
M=\frac{i k}{2 \pi \sqrt{3}} \int_{0}^{\pi / 2}\left(\cos (\sigma) \tilde{\psi}_{0}(\cos (\sigma))-\psi_{0}(\cos (\sigma))\right) \sigma d \sigma
$$

Once $\rho$ is computed, $T$ can be computed by $(2 \mathrm{~b})$.

\section{References}

[1] Bierman, G. J., A particular class of singular integral equations, Siam J. Appl. Math. 20, 99-109 (1971).

[2] Bueckner, H. F., On a class of singular integral equations, J. Math. Anal. Appl. 14, 392-426 (1966).

[3] Carrier, G. F., Krook, M., and Pearson, C. E., Functions of a Complex Variable (McGraw-Hill, New York, 1966).

[4] Latta, G. E., Transform methods, Handbook of Applied Mathematics (Chap. 11), C. E. Pearson, Ed. (Van Nostrand Reinhold Co., New York, 1974), pp. 635-636.

[5] Levinson, N., Simplified treatment of integrals of Cauchy type, the Hilbert problem and singular integral equations, Siam Review, 7, 474-497 (1965).

[6] Marcuvitz, N., Ed., Waveguide Handbook (Chap. 5) (McGraw-Hill, New York, 1957).

[7] Muskhelishvili, N. I., Singular Integral Equations, Translated from 1946 Russian-language edition, Trans. Ed. by J. R. M. Radok (P. Noordhoff N. V., Gronigen, Holland, 1953).

[8] Pennline, J. A., A class of singular integral equations, Ph. D. dissertation, University of Virginia, Charlottesville, Virginia, 1974.

[9] Schwinger, J., and Saxon, D., Discontinuities in Waveguides (Chap. 5) (Gordon and Breach Science Publishers, Ltd., 1968).

[10] Vekua, N. P., Systems of Singular Integral Equations, Translated by A. G. Gibbs and G. M. Simmons from Russian-language edition, Trans. Ed. by J. H. Fersigner (P. Noordhoff Ltd., Gronigen, the Netherlands, 1967).

(Paper 80B3-452) 